\title{
A Dynamic Output Feedback Controller for NCS based on Delay Estimates *
}

\author{
Marc Jungers, ${ }^{\text {a,b }}$ Eugênio B. Castelan, ${ }^{\mathrm{c}}$ Vitor M. Moraes, ${ }^{\mathrm{c}}$ Ubirajara F. Moreno ${ }^{\mathrm{c}}$ \\ ${ }^{a}$ CNRS, CRAN, UMR 7032, Vandoeuvre-les-Nancy, F-54516, France (email: marc.jungers@univ-lorraine.fr) \\ ${ }^{\mathrm{b}}$ Université de Lorraine, CRAN, UMR 7032, Vandoeuvre-les-Nancy, F-54516, France \\ ${ }^{\mathrm{c}} D A S-C T C-U F S C$ \\ P.O. Box 476, 88040-900 Florianópolis, SC, Brazil \\ (emails: [eugenio,rattus,moreno]@das.ufsc.br)
}

\begin{abstract}
The stabilization of a network controlled system including a global time-varying delay is investigated in this note. This delay is considered to be unknown but it is assumed that a bounded error estimate is available. The exponential uncertainty induced by the time-varying delay is decomposed into a sum of a polytopic term and an uncertain bounded term. Sufficient conditions to design a dynamic output feedback controller depending on estimate of the time-varying delay are proposed as LMIs. An illustration shows how our methodology enlarges the design techniques of the literature.
\end{abstract}

Key words: Dynamic Output Feedback, Network Control System, Delay estimate.

\section{Introduction}

The Network Controlled Systems (NCS) are frequently encountered in practice for widespread fields of applications due to their suitable and flexible structure $[1,3]$. Nevertheless some aspects of NCS such as time-varying delays, quantization, drop-out, imply that the stability analysis and control design become fundamentally more difficult to investigate [7]. They lead to a rich literature in automatic control particularly in what concerns the stability aspects of systems with time-varying delays $[5,9,11,23,24]$.

In order to achieve stability requirements, controller synthesis methodologies have been proposed. Among of them the use of Lyapunov functions is the most popular for the stability analysis [5,23] and control synthesis [6]. Robust controllers independent on the time-varying delays may be designed to stabilize the NCS but such strategies are generally source of conservatism. In order to reduce such a conservatism for NCS for which the current time-varying delay is available, controllers depending on the time-varying delays may be proposed.

\footnotetext{
^ This paper was not presented at any IFAC meeting. Corresponding author M. Jungers. Tel. +33 (0)3 83595704 . Fax $+33(0) 383595644$.
}

Recently output dynamic feedback design for NCS has been proposed using Linear Matrix Inequalities (LMI), based on the design procedure introduced by C. Scherer in [21] or on the elimination lemma. The output dynamic feedback may be independent with respect to the timevarying delay $[8,10,18]$, or time-varying delay dependent: [22] considers two delays (back and forth), [17] a global time-varying delay less than the sampling period and [16] a global and bounded time-varying delay multiple of the sampling period.

Knowing the value of the current time-varying delay is in practice difficult, not to say impossible. Nevertheless tools allowing to have a time-varying delay estimate are provided in the literature (see for more details $[4,20]$ ). The goal of this paper is not to enrich this mature literature, but to make the best use of the information conveyed by the time-varying delay estimate in the stabilizing controller design. Such an idea was investigated in [13] for designing a state-feedback controller dependent on this estimate by considering an additional uncertain term in the exponential uncertainty.

This note aims at extending the results in [13] by relaxing the assumption of the state availability and by proposing a dynamic output feedback controller dependent on an estimate of the time-varying delay. The main result 
is allowed by considering the exponential uncertainty related to the estimate of the time-varying delay decomposed into a polytopic term and an uncertain term. Via the Petersen's Lemma and changes of variables similar to the one proposed by C. Scherer, we obtain sufficient conditions formulated as LMIs leading to the controller design ensuring the closed-loop stability of the NCS.

The note is organized as follows. In Section 2, the problem of dynamic output feedback controller design dependent on an estimate of the time-varying delay is formulated. Some preliminaries are proposed in Section 3 to allow the proof of the main result presented in Section 4. A numerical illustration is given in Section 5 to highlight the efficiency of our result compared with the literature, before some concluding remarks in Section 6 .

Notation. (. $)^{\prime}$ and $\star$ denote respectively the transpose and the symmetric block in a symmetric matrix. $\operatorname{diag}(A, B)$ denotes the diagonal matrix composed by matrices $A$ and $B$. The hermitian operator is given by $\mathrm{He}(M)=M+M^{\prime}$, for any square matrix $M . \sigma_{\max }(M)$ denotes the maximum singular value of the matrix $M$. Matrices $I_{n \times m}$ and $0_{n \times m}$ are respectively Identity matrix of size $n \times m$ and the null matrix of size $n \times m$. The shortcuts $I_{n}=I_{n \times n}$ and $0_{n}=0_{n \times n}$ will be used. The symbol $\bullet$ denotes an irrelevant block in a matrix.

\section{Problem Description}

Consider the following continuous-time system,

$\begin{aligned} \dot{x}(t) & =A x(t)+B u(t), \quad \forall t \geq 0, \\ y(t) & =C x(t),\end{aligned}$

where $x(t) \in \mathbb{R}^{n}, u(t) \in \mathbb{R}^{m}$ and $y(t) \in \mathbb{R}^{p}$ are respectively the continuous-time state, input and output of the system. The state $x(t)$ is sampled with a sampling period $T$ to obtain the sampling states $x_{k}=x(k T)$ at time $k T$, $(k \in \mathbb{N})$. Due to the NCS structure, the continuous-time input $u(t)$ applied to the system is delayed by a global time-varying delay $\tau_{k}$ which is assumed to be unknown and to verify $0 \leq \tau_{k} \leq T$, that is,

$$
u(t)= \begin{cases}u_{k-1}, & \forall t \in\left[k T, k T+\tau_{k}\right), \\ u_{k}, & \forall t \in\left[k T+\tau_{k},(k+1) T\right) .\end{cases}
$$

Remark 1 The assumption $0 \leq \tau_{k} \leq T$ may be relaxed into $0 \leq \tau_{k} \leq \ell T$, with $\ell$ an integer greater than one by an event-based approach [12] or into $(\ell-1) T \leq \tau_{k} \leq \ell T$ by using an extended state [24]. For the sake of clarity, we maintain in the sequel the assumption that the delays are lower than the sampling interval. Furthermore, this assumption is in accordance with NCS with real-time requirements, where reliable network environment with deterministic protocols are used.
In such a framework, the discrete-time system writes

$$
\begin{aligned}
x_{k+1} & =A_{\mathrm{d}} x_{k}+\Omega\left(\tau_{k}\right) B u_{k}+\left(B_{\mathrm{d}}-\Omega\left(\tau_{k}\right) B\right) u_{k-1}, \\
y_{k} & =C x_{k},
\end{aligned}
$$

where $A_{\mathrm{d}}=e^{A T}, B_{\mathrm{d}}=\int_{0}^{T} e^{A s} \mathrm{~d} s B$ and $\Omega(\tau)=$ $\int_{0}^{T-\tau} e^{A s} \mathrm{~d} s$, with $\tau \in[0, T]$. By introducing the extended state $\eta_{k}=\left[\begin{array}{c}x_{k} \\ u_{k-1}\end{array}\right] \in \mathbb{R}^{n+m}$, one gets by following [13] the reformulation of the system (4):

$$
\begin{aligned}
\eta_{k+1} & =\bar{A}\left(\tau_{k}\right) \eta_{k}+\bar{B}\left(\tau_{k}\right) u_{k}, \\
y_{k} & =\bar{C} \eta_{k},
\end{aligned}
$$

where $\bar{A}\left(\tau_{k}\right)=\left[\begin{array}{cc}A_{\mathrm{d}} & \left(B_{\mathrm{d}}-\Omega\left(\tau_{k}\right) B\right) \\ 0_{m \times n} & 0_{m}\end{array}\right] ; \bar{B}\left(\tau_{k}\right)=$
$\left[\begin{array}{c}\Omega\left(\tau_{k}\right) B \\ I_{m}\end{array}\right] ; \bar{C}=\left[\begin{array}{ll}C & 0_{p \times m}\end{array}\right]$.

As it has been mentioned, the current delay $\tau_{k}$ is not exactly known and we consider the following assumption.

Assumption 2 An estimate $\hat{\tau}_{k} \in[0, T]$ of $\tau_{k}$ is available and is characterized by $\delta \tau_{\text {min }} \leq \delta \tau_{k}=\tau_{k}-\hat{\tau}_{k} \leq \delta \tau_{\text {max }}$, where by definition $-T \leq \delta \tau_{\min } \leq \delta \tau_{\max } \leq T$.

We thus introduce the reformulation

$$
\begin{aligned}
\eta_{k+1}=\left(\bar{A}\left(\hat{\tau}_{k}\right)+\right. & {\left.\left[\begin{array}{cc}
0_{n} & -W_{k} B \\
0_{m \times n} & 0_{m}
\end{array}\right]\right) \eta_{k} } \\
& +\left(\bar{B}\left(\hat{\tau}_{k}\right)+\left[\begin{array}{c}
W_{k} B \\
0_{m}
\end{array}\right]\right) u_{k}
\end{aligned}
$$

where $W_{k}=\Omega\left(\tau_{k}\right)-\Omega\left(\hat{\tau}_{k}\right)=\Omega\left(T-\delta \tau_{k}\right)\left[I_{n}+A \Omega\left(\hat{\tau}_{k}\right)\right]$. Moreover, we relax the state availability assumption in [13] and assume here what follows.

Assumption 3 Only the output $y_{k}$ of the system is available, instead of its state $x_{k}$.

The paper aims at solving the following problem.

Problem 4 Consider the system (8). Under Assumptions 2 and 3, determine a full order dynamic output feedback, in the form

$$
\begin{aligned}
\zeta_{k+1} & =A_{c}\left(\hat{\tau}_{k}\right) \zeta_{k}+B_{c}\left(\hat{\tau}_{k}\right) y_{k}, \\
u_{k} & =C_{c} \zeta_{k}+D_{c} y_{k},
\end{aligned}
$$

where $\zeta_{k} \in \mathbb{R}^{n+m}$, which asymptotically stabilizes the system (8). 
Remark 5 The model (10) involves only constant matrices $C_{c}$ and $D_{c}$. It allows to consider in the sequel a structure of the output dynamic feedback which is linear with respect to the polytopic weighting gains.

\section{Preliminaries}

By defining $0 \leq \rho_{k}=1-\frac{\tau_{k}}{T} \leq 1$, we can decompose $\Omega\left(\tau_{k}\right)$ into an uncertain polynomial parameter dependent matrix and an uncertain bounded remainder $\Delta \Omega_{k}$ : $\Omega\left(\tau_{k}\right)=T \int_{0}^{\rho_{k}} e^{A T s^{\prime}} \mathrm{d} s^{\prime}=\sum_{i=1}^{h} \frac{A^{i-1} T^{i}}{i !} \rho_{k}^{i}+\Delta \Omega_{k}$. Let introduce the following lemma, proven in [14].

Lemma 6 The uncertain polynomial parameter dependent matrix $L(\rho)=L_{0}+\rho L_{1}+\rho^{2} L_{2}+\cdots+\rho^{h} L_{h}$, with $0 \leq \rho \leq 1$, can be rewritten as a convex polytope with $h+1$ vertices, with $\mathcal{H}=\{1 ; \cdots ;(h+1)\}$ : $L(\rho)=\sum_{i \in \mathcal{H}} \mu_{i}(\rho) V_{i}$, with $V_{i}=\sum_{j=0}^{i-1} L_{j} ; \forall i \in \mathcal{H}$ and $\mu_{h+1}=\rho^{h} ; \mu_{i}=\rho^{i-1}(1-\rho), \forall i \in\{1, \cdots, h\}$, which verify $\sum_{i \in \mathcal{H}} \mu_{i}(\rho)=1$, and $\mu_{i}(\rho) \geq 0, \forall i \in \mathcal{H}$.

By identifying $L_{0}=0_{n}$; and $L_{i}=\frac{A^{i-1} T^{i}}{i !}, \forall i=$ $1, \cdots, h$, we have, with the definition of $\dot{V}_{i}$ given in Lemma 6: $\Omega\left(\tau_{k}\right)=\sum_{i \in \mathcal{H}} \mu_{i}\left(\hat{\rho}_{k}\right) V_{i}+\left(W_{k}+\Delta \Omega_{k}\right)$, and the following decompositions

$$
\begin{gathered}
\bar{A}\left(\tau_{k}\right)=\sum_{i \in \mathcal{H}} \mu_{i}\left(\hat{\rho}_{k}\right) \bar{A}_{i}+\left[\begin{array}{c}
I_{n} \\
0_{m \times n}
\end{array}\right]\left(W_{k}+\Delta \Omega_{k}\right)\left[0_{n}-B\right] ; \\
\bar{B}\left(\tau_{k}\right)=\sum_{i=1}^{h+1} \mu_{i}\left(\hat{\rho}_{k}\right) \bar{B}_{i}+\left[\begin{array}{c}
I_{n} \\
0_{m \times n}
\end{array}\right]\left(W_{k}+\Delta \Omega_{k}\right) B ; \\
\bar{A}_{i}=\left[\begin{array}{cc}
A_{\mathrm{d}} & \left(B_{\mathrm{d}}-V_{i} B\right) \\
0_{m \times n} & 0_{m}
\end{array}\right] ; \bar{B}_{i}=\left[\begin{array}{c}
V_{i} B \\
I_{m}
\end{array}\right] .
\end{gathered}
$$

In order to cope with the time-varying term $\left(W_{k}+\Delta \Omega_{k}\right)$ in the decomposition of $\Omega\left(\tau_{k}\right)$, we remind the following lemma provided in [19].

Lemma 7 Given any positive scalar $\lambda>0$ and any $m a-$ trix $\Gamma$ such that $\Gamma^{\prime} \Gamma \leq \gamma^{2} I$, for any matrices $H$ and $Q$ of appropriated dimensions, we have $H \Gamma Q+Q^{\prime} \Gamma^{\prime} H^{\prime}+$ $\lambda H H^{\prime}+\gamma^{2} \frac{Q^{\prime} Q}{\lambda} \geq 0$.

The main idea is to consider for the dynamic output feedback controller a polytopic structure depending on the parameters $\mu_{i}\left(\hat{\rho}_{k}\right)$, with $\hat{\rho}_{k}=1-\frac{\hat{\tau}_{k}}{T}$. That is we impose $A_{\mathrm{c}}\left(\hat{\tau}_{k}\right)=\sum_{i \in \mathcal{H}} \mu_{i}\left(\hat{\rho}_{k}\right) A_{\mathrm{c}, i} ; B_{\mathrm{c}}\left(\hat{\tau}_{k}\right)=\sum_{i \in \mathcal{H}} \mu_{i}\left(\hat{\rho}_{k}\right) B_{\mathrm{c}, i}$.
The controlled system, with state $z_{k}=\left[\begin{array}{c}\eta_{k} \\ \zeta_{k}\end{array}\right]$ becomes

$$
z_{k+1}=\sum_{i=1}^{h+1} \mu_{i}\left(\hat{\rho}_{k}\right)\left(\mathbb{A}_{i}+E \Gamma_{k} F\right) z_{k}=\mathbb{A}_{\mathrm{cl}, k} z_{k},
$$

where $F=\left[\begin{array}{lll}0_{n}-B & 0_{n \times(n+m)}\end{array}\right]+\left[\begin{array}{lll}B D_{\mathrm{c}} \bar{C} & B C_{\mathrm{c}}\end{array}\right]$;

$$
\begin{gathered}
\mathbb{A}_{i}=\left[\begin{array}{cc}
\bar{A}_{i}+\bar{B}_{i} D_{\mathrm{c}} \bar{C} & \bar{B}_{i} C_{\mathrm{c}} \\
B_{\mathrm{c}, i} \bar{C} & A_{\mathrm{c}, i}
\end{array}\right] ; E=\left[\begin{array}{c}
I_{n} \\
0_{(n+2 m) \times n}
\end{array}\right] ; \\
\Gamma_{k}=W_{k}+\Delta \Omega_{k}=\Omega\left(\tau_{k}\right)-\Omega\left(\hat{\tau}_{k}\right)+\Delta \Omega_{k} .
\end{gathered}
$$

The equation (11) exhibits a polytopic part and an uncertain part, where $W_{k}$ and $\Delta \Omega_{k}$ are gathered in $\Gamma_{k}$. We should point out that there exists an upper bound of the maximal singular value of matrix $\Gamma_{k}=\Omega\left(T-\delta \tau_{k}\right)\left[I_{n}+\right.$ $\left.A \Omega\left(\hat{\tau}_{k}\right)\right]+\Delta \Omega_{k}$. It has been already shown in [13] that the uncertainty $\Omega\left(T-\delta \tau_{k}\right)$ is norm-bounded on a compact $\delta \tau_{k} \in\left[\delta \tau_{\min } ; \delta \tau_{\max }\right]$. The matrix $\Delta \Omega_{k}$ is also bounded on the range $0 \leq \hat{\rho}_{k} \leq 1$. Thus there exists a scalar $\gamma$ such that, with $\overline{\mathcal{T}}\left(\tau_{k}\right)=\left[\tau_{k}-\delta \tau_{\max } ; \tau_{k}-\delta \tau_{\min }\right] \cap[0 ; T]$

$$
\sup _{\tau_{k} \in[0 ; T] ; \hat{\tau}_{k} \in \mathcal{T}\left(\tau_{k}\right)} \sigma_{\max }\left(\Gamma_{k}\right) \leq \gamma
$$

Remark 8 We can obtain numerically an upper bound of the maximal singular value of $\Gamma_{k}$ by computing offline the singular value of $\Gamma_{k}$ on a sufficiently tight grid verifying $\tau_{k} \in[0 ; T]$ and $\hat{\tau}_{k} \in \mathcal{T}\left(\tau_{k}\right)$.

The fundamental preliminary result is presented in the following proposition.

Proposition 9 If there exist a matrix $U \in \mathbb{R}^{2(n+m) \times 2(n+m)}$, symmetric matrices $S_{i} \in \mathbb{R}^{2(n+m) \times 2(n+m)}$, matrices $A_{c, i}, B_{c, i},(i \in \mathcal{H}), C_{c}$ and $D_{c}$ such that the inequality

$$
\left[\begin{array}{cc}
U+U^{\prime}-S_{i} & \star \\
\left(\mathbb{A}_{i}+E \Gamma_{k} F\right) U & S_{j}
\end{array}\right]>0_{4(n+m)}, \forall(i, j) \in \mathcal{H} \times \mathcal{H}
$$

is verified, then the controller (9)-(10) stabilizes the NCS (8).

Proof 10 Assuming that the inequality (13) is verified, this implies that $U+U^{\prime}-S_{i}>0_{2(n+m)}$ and $S_{i}>0_{2(n+m)}$, which induces that $U$ is of full rank and invertible. Moreover $\left(U-S_{i}\right)^{\prime} S_{i}^{-1}\left(U-S_{i}\right) \geq 0_{2(n+m)}$ allows the inequality, $\forall(i, j) \in \mathcal{H} \times \mathcal{H}$

$$
\left[\begin{array}{cc}
U^{\prime} S_{i}^{-1} U & \star \\
\left(\mathbb{A}_{i}+E \Gamma_{k} F\right) U & S_{j}
\end{array}\right]>0_{4(n+m)} .
$$


By pre-multiplying the inequality (14) by diag $\left(\left(U^{\prime}\right)^{-1} ; S_{i}^{-1}\right)$, and by post-multiplying it by $\operatorname{diag}\left(U^{-1} ; S_{i}^{-1}\right)$, we have, with the change of variables $P_{i}=S_{i}^{-1}, \mathcal{L}_{i j}=$ $\left[\begin{array}{cc}P_{i} & \star \\ P_{j}\left(\mathbb{A}_{i}+E \Gamma_{k} F\right) & P_{j}\end{array}\right]>0_{4(n+m)}, \forall(i, j) \in \mathcal{H} \times \mathcal{H}$.

By denoting $P\left(\hat{\tau}_{k}\right)=\sum_{i \in \mathcal{H}} \mu_{i}\left(\hat{\rho}_{k}\right) P_{i}$ and by summing the last inequality and finally applying a Schur complement, one gets

$$
\mathbb{A}_{c l, k}^{\prime} P\left(\hat{\tau}_{k+1}\right) \mathbb{A}_{c l, k}-P\left(\hat{\tau}_{k}\right)<0_{2(n+m)}
$$

The positive definiteveness of the matrices $P_{i}$ and the inequality (15) allow to prove that the function $V\left(z_{k}, \hat{\tau}_{k}\right)=$ $z_{k}^{\prime} P\left(\hat{\tau}_{k}\right) z_{k}$ is a Lyapunov function for the closed-loop system (11), which is thus asymptotically stable.

The next section will provide sufficient conditions designing the dynamic output feedback (9).

\section{Designing the dynamic output feedback con- troller}

The inequality (13) is not linear with respect to the gain matrices of the controller, but via a change of variables, originally proposed in an LTI case in [21], this is reformulated into LMIs.

Proposition 11 Let us consider the predefined system (8) and a scalar $\gamma$ verifying the inequality (12). Assume that there exists matrices $X, Y, K \in \mathbb{R}^{(n+m) \times(n+m)}$, matrices $\hat{A}_{i} \in \mathbb{R}^{(n+m) \times(n+m)}, \hat{B}_{i} \in \mathbb{R}^{(n+m) \times p}$, matrices $\hat{C} \in \mathbb{R}^{m \times(n+m)}$ and $\hat{D} \in \mathbb{R}^{m \times p}$ and symmetric positive definite matrices $\hat{S}_{i} \in \mathbb{R}^{2(n+m) \times 2(n+m)},(i \in \mathcal{H})$ such that the following LMIs are verified, $\forall(i, j) \in \mathcal{H} \times \mathcal{H}$

$$
\left[\begin{array}{cccc}
\mathcal{X}_{1}-\hat{S}_{i} & \star & \star & \star \\
\mathcal{X}_{2, i} & \hat{S}_{j} & \star & \star \\
0_{n \times 2(n+m)} & \mathcal{X}_{3} & I_{n} & \star \\
\gamma \mathcal{X}_{4} & 0_{n \times 2(n+m)} & 0_{n} & I_{n}
\end{array}\right]>0_{(6 n+4 m)},
$$

where

$$
\begin{aligned}
\mathcal{X}_{1}= & {\left[\begin{array}{cc}
Y+Y^{\prime} & I_{(n+m)}+K \\
I_{(n+m)}+K^{\prime} & X+X^{\prime}
\end{array}\right] } \\
\mathcal{X}_{2, i}= & {\left[\begin{array}{cc}
Y^{\prime} \bar{A}_{i}+\hat{B}_{i} \bar{C} & \hat{A}_{i} \\
\bar{A}_{i}+\bar{B}_{i} \hat{D} \bar{C} & \bar{A}_{i} X+\bar{B}_{i} \hat{C}
\end{array}\right] ; } \\
\mathcal{X}_{3}= & {\left.\left[\begin{array}{cc}
I_{n} & 0_{n \times m}
\end{array}\right] Y\left[\begin{array}{ll}
I_{n} & 0_{n \times m}
\end{array}\right]\right] } \\
\mathcal{X}_{4}= & {\left[\begin{array}{ll}
0_{n} & -B
\end{array}\right]\left[\begin{array}{ll}
I_{(n+m)} & X
\end{array}\right]+\left[\begin{array}{ll}
B \hat{D} \bar{C} & B \hat{C}
\end{array}\right] . }
\end{aligned}
$$

By choosing any invertible matrix $M \in \mathbb{R}^{(n+m) \times(n+m)}$, let us set the matrix $N=\left(M^{\prime}\right)^{-1}\left(K-Y^{\prime} X\right) . N$ is invertible and the controller with gain matrices

$$
\begin{aligned}
D_{c}= & \hat{D} \\
C_{c}= & (\hat{C}-\hat{D} \bar{C} X) N^{-1} \\
B_{c, i}= & \left(M^{\prime}\right)^{-1}\left(\hat{B}_{i}-Y^{\prime} \bar{B}_{i} \hat{D}\right) \\
A_{c, i}= & \left(M^{\prime}\right)^{-1}\left(\hat{A}_{i}-Y^{\prime}\left(\bar{A}_{i}+\bar{B}_{i} \hat{D} \bar{C}\right) X\right. \\
& \left.\quad-Y^{\prime} \bar{B}_{i} C_{c} N-M^{\prime} B_{c, i} \bar{C} X\right) N^{-1},
\end{aligned}
$$

stabilizes the closed-loop system (11).

Proof 12 The proof is based on a suitable congruence transformation and a change of variables allowing to obtain the inequality (13) in Proposition 9. Such a transformation has been initially proposed in [21] and extended in [2,15]. The time-dependency of the Lyapunov function implies that the decomposition provided in [21] should be applied on the multiplier matrix $U$ instead of the matrices $S_{i}(i \in \mathcal{H})$ and that $U$ should be timeindependent. Let us decompose the matrices $U$ and $U^{-1}$ as $U=\left[\begin{array}{l}X \bullet \\ N \bullet \bullet\end{array}\right] ; U^{-1}=\left[\begin{array}{ll}Y \bullet \\ M \bullet \bullet\end{array}\right]$. The matrix $M \in$ $\mathbb{R}^{(n+m) \times(n+m)}$ appearing $U^{-1}$ is assumed to be invertible. This invertibility property will be induced thereafter by the constraints about matrix $U$. Let us introduce the matrix $\Pi=\left[\begin{array}{cc}Y & I_{(n+m)} \\ M & 0_{(n+m)}\end{array}\right]$. We have the following relations $U \Pi=\left[\begin{array}{ll}I_{(n+m)} & X \\ 0_{(n+m)} & N\end{array}\right] ; \Pi^{\prime} U \Pi=\left[\begin{array}{cc}Y^{\prime} & K \\ I_{(n+m)} & X\end{array}\right]$; by noting $K=\left(Y^{\prime} X+M^{\prime} N\right)$, as introduced in the proposition. Using the structure of $U$ allows to reformulate $\Pi^{\prime}\left(U+U^{\prime}\right) \Pi=\mathcal{X}_{1} ; \Pi^{\prime} \mathbb{A}_{i} U \Pi=\mathcal{X}_{2, i} ; E^{\prime} \Pi=\mathcal{X}_{3}$; $F U \Pi=\mathcal{X}_{4}$ where the changes of variables are used

$$
\begin{aligned}
\hat{D} & =D_{c} ; \\
\hat{C} & =\hat{D} \bar{C} X+C_{c} N ; \\
\hat{B}_{i}= & M^{\prime} B_{c, i}+Y^{\prime} \bar{B}_{i} \hat{D} ; \\
\hat{A}_{i}= & M^{\prime} A_{c, i} N+Y^{\prime}\left(\bar{A}_{i}+\bar{B}_{i} \hat{D} \bar{C}\right) X \\
& \quad+Y^{\prime} \bar{B}_{i} C_{c} N+M^{\prime} B_{c, i} \bar{C} X .
\end{aligned}
$$

In addition, we will use in the sequel the change of variables $\hat{S}_{i}=\Pi^{\prime} S_{i} \Pi$. By applying the Schur complement to the LMI (16) and due Lemma 7 , we have $(\lambda=1)$,

$$
\left[\begin{array}{cc}
\Pi^{\prime}\left(U+U^{\prime}-S_{i}\right) \Pi \quad & \star \\
\Pi^{\prime}\left(\mathbb{A}_{i}+E \Gamma_{k} F_{i}\right) U \Pi \Pi^{\prime} S_{j} \Pi
\end{array}\right]>0_{4(n+m)} .
$$

By considering $M$ invertible, the matrix $\Pi$ is invertible. Pre-multiplying the inequality (29) by $\operatorname{diag}\left(\left(\Pi^{\prime}\right)^{-1},\left(\Pi^{\prime}\right)^{-1}\right)$ 
and post-multiplying it by $\operatorname{diag}\left(\Pi^{-1}, \Pi^{-1}\right)$ leads to inequality (13). We have only to prove that $N$ defined by $N=\left(M^{\prime}\right)^{-1}\left(K-Y^{\prime} X\right)$ is invertible to allow the change of variables in Equations (22) and (24) and to end the proof. Due to Inequality (16), $\mathcal{X}_{1}$ is invertible and also

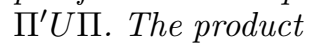

$$
\left[\begin{array}{cc}
I_{(n+m)} & -Y^{\prime} \\
0_{(n+m)} & I_{(n+m)}
\end{array}\right]\left[\begin{array}{cc}
Y^{\prime} & K \\
I_{(n+m)} & X
\end{array}\right]=\left[\begin{array}{cc}
0_{(n+m)} & K-Y^{\prime} X \\
I_{(n+m)} & X
\end{array}\right]
$$

is then invertible, as $\left(K-Y^{\prime} X\right)$ and $N$.

The proof of Proposition 11 considers the particular case $\lambda=1$ in Lemma 7 . In fact setting $\lambda=1$ is with no loss of generality but allows the linearization of the condition (16). The main result provided by Proposition 11 is based on the feasibility of LMIs (16) for a fixed value of $\gamma$. The question then arises to know what range of uncertainty $\left[\delta \tau_{\max } ; \delta \tau_{\min }\right]$ for the dynamic output feedback controller ensures the closed-loop stability.

Proposition 13 Considering that LMIs (16) are feasible for a value $\gamma=\gamma^{*}>0$, then they are feasible for any value $0<\gamma \leq \gamma^{*}$.

Proof 14 The property is obvious by applying on LMIs (16), with $\gamma=\gamma^{*}>0$, the change of basis $\operatorname{diag}\left(I_{5 n+4 m} ;\left(\gamma^{*}\right)^{-1} I_{n}\right)$.

Based on Proposition 13, a numerical line-search allows to compute a threshold $\gamma^{*}$ of LMIs (16)-feasibility. By defining $\delta=\delta \tau_{\max }=-\delta \tau_{\min }$, an abacus based on the increasing function $\delta \rightarrow \gamma(\delta)$ can be computed. This abacus is thereafter used to obtain $\delta=\delta^{*}$ related to $\gamma^{*}$.

\section{Illustration}

In this section, we consider an example coming from [6] and revisited in [13], for which $n=2, m=1, A=$ $\left[\begin{array}{cc}103.5 & 0 \\ 0 & -43.5\end{array}\right], B=\left[\begin{array}{c}33.6 \\ -5.1\end{array}\right]$. It has been pointed out that the design of a delay-independent state-feedback controller by applying the design method proposed by [6] failed with $T=0.005$, nevertheless a design of a statefeedback controller dependent on an estimate of the delay with $\delta=0.0015=3 T / 10$ succeeded via the method provided in [13]. Nevertheless such a method is not suitable when the state is not fully accessible. Let us consider $C=\left[\begin{array}{ll}10 & 1\end{array}\right]$ to define the output of the system. $\gamma$ is given in Table 1 , as function of $h$. $h$ should be chosen to reach a compromise between the improvement of $\gamma$ and the size of the LMIs in Proposition 11. Here we choose $h=1$ to apply Proposition 11, which leads to a solution.

By considering $\delta=\delta \tau_{\max }=-\delta \tau_{\min }$, one can plot the function $\delta \rightarrow \gamma(\delta)$ for distinct values of $h$ on Figure 1 .

\begin{tabular}{|c||c|c|c|c|c|}
\hline$h$ & 1 & 2 & 3 & 4 & 5 \\
\hline$\gamma$ & 0.0039 & 0.0026 & 0.0024 & 0.0023 & 0.0023 \\
\hline
\end{tabular}

Table 1

Upper bound $\gamma$ associated with $\Gamma_{k}$ in function of $h$.

For a fixed value of $\delta$, when $h$ increases, the upper bound of the uncertainty decreases by definition. For our simu-

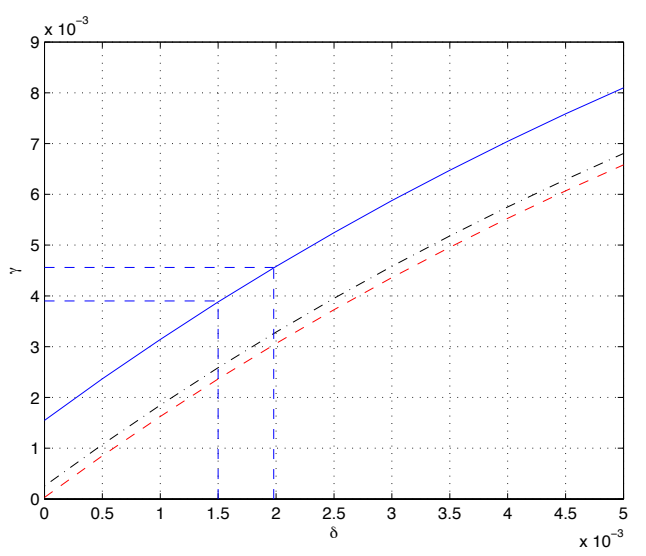

Fig. 1. Values of the bound $\gamma$ in function of $\delta=\delta \tau_{\max }=-\delta \tau_{\min } \in[0 ; T]$ for $h=1$ (blue solid line), $h=2$ (black dashdot line) and $h=3$ (red dashed line).

lation, the time-varying delay $\tau_{k}$ is randomly generated in $[0, T]$ and $\hat{\tau}_{k}$ in $\mathcal{T}\left(\tau_{k}\right)$. When choosing $M=I_{(n+m)}$, our approach leads to the state- and input-trajectories plotted in Figure 2, converging to the origin. Via the zero order holder, the continuous time system is simulated via TrueTime on Matlab-Simulink. The resulting continuous-time trajectories are superposed on the Figure 2. A numerical line-search allows to determine the
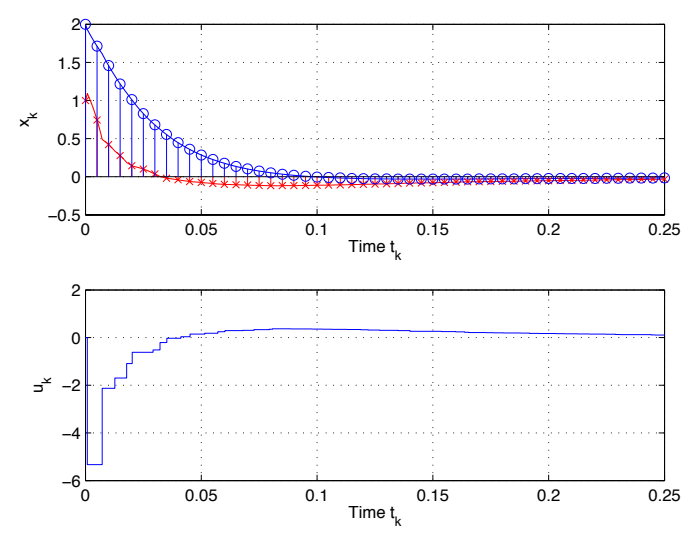

Fig. 2. State $x_{k}\left(\times\right.$ for $x_{1, k}$ and $\circ$ for $\left.x_{2, k}\right)$ and the input $u_{k}$, in function of time $t_{k}$.

thresholds $\gamma^{*}$ in function of $h$ (given in Table 2). By using Figure 1, we can assert that our designed dynamic output feedback stabilizes the system for an estimate error less than $\pm 1.98 \times 10^{-3} s$. 


\begin{tabular}{|c||c|c|c|}
\hline$h$ & 1 & 2 & 3 \\
\hline$\gamma^{*}$ & $4.56 \times 10^{-3}$ & $4.32 \times 10^{-3}$ & $4.28 \times 10^{-3}$ \\
\hline
\end{tabular}

Table 2

Threshold $\gamma^{*}$ of LMIs (16)-feasibility in function of $h$.

\section{Conclusion}

The design of a dynamic output feedback controller for a network controlled system based on the knowledge of time-varying delay estimate has been studied in this note. As a preliminary result, the exponential uncertainty is rewritten into a sum of a polytopic term and an uncertain bounded term. With the help of the Petersen's lemma and a change of variables inspired by the Scherer's one, a sufficient condition formulated as LMIs has been provided to design a dynamic output feedback controller stabilizing the network controlled system. A illustrative example has been proposed.

\section{Acknowledgements}

E. B. Castelan has a financial support by CNPq Brazil. This work was partially supported by the project PICS CNRS $n^{\circ}$ 5284, by CAPES-COFECUB Project $n^{\circ} 701 / 11$ and by ANR project ArHyCo, Programme "Systèmes Embarqués et Grandes Infrastructures" ARPEGE, contract number ANR-2008 SEGI 004 0130011459 and finally by the European Community's Seventh Framework Programme (FP7/2007-2013) under grant agreement $\mathrm{n}^{\circ}$ 257462: HYCON2 Network of Excellence "Highly-Complex and Networked Control Systems".

\section{References}

[1] P. Antsaklis and J. Baillieul. Special issue on technology of networked control systems. Proceedings of the IEEE, 95(1):58, 2007.

[2] P. Apkarian and R. J. Adams. Advanced gain-scheduling techniques for uncertain systems. IEEE Transactions on Control System Technology, 6(1):21-32, 1998.

[3] K. J. Åström and B. Wittenmark. Computer-Controlled Systems. Prentice Hall, 1997.

[4] G. C. Carter, editor. Time delay estimation, volume ASSP29. IEEE Transactions on Acoustics, Speech and Signal Processing, 1981.

[5] M. B. G. Cloosterman, N. van de Wouw, W. P. M. H. Heemels, and H. Nijmeijer. Stability of networked control systems with uncertain time-varying delays. IEEE Transaction on Automatic Control, 54(7):1575-1580, 2009.

[6] M.B.G. Cloosterman, L. Hetel, N. van de Wouw, W.P.M.H. Heemels, J. Daafouz, and H. Nijmeijer. Controller synthesis for networked control systems. Automatica, 46:1584-1594, 2010.

[7] M. C. F. Donkers, W. P. M. H. Heemels, N. van de Wouw, and L. Hetel. Stability analysis of networked control systems using a switched linear systems approach. IEEE Transactions on Automatic Control, 56(9):2101-2115, 2011.
[8] L. Dritsas and A. Tzes. Robust output feedback control of networked systems. In Proceedings of the European Control Conference, pages 3939-3945, Kos, Greece, July 2007.

[9] L. Dritsas and A. Tzes. Robust stability analysis of networked systems with varying delays. International Journal of Control, 82(12):2347-2355, December 2009.

[10] H. Fujioka. Stability analysis for a class of networked/ embedded control systems: output feedback case. In Proceedings of the 17th IFAC World Congress, pages 42104215, Seoul, Korea, 2008.

[11] J. Hespanha, P. Naghshtabrizi, and Y. Xu. A survey of recent results in networked control systems. IEEE Special Issue on Technology of Networked Control Systems, 95(1):138-162, January 2007.

[12] L. Hetel, J. Daafouz, and C. Iung. Analysis and control of LTI and switched systems in digital loops via an event-based modeling. International Journal of Control, 81(7):1125-1138, July 2008.

[13] L. Hetel, J. Daafouz, J.-P. Richard, and M. Jungers. Delaydependant sampled-data control based on delay estimates. Systems \& Control Letters, 60:146-150, 2011.

[14] M. Jungers, L. Hetel, and J. Daafouz. Guaranteed cost for control of networked control systems with small time-varying delays. In 19th International Symposium on Mathematical Theory of Networks and Systems (MTNS), pages 1465-1470. Budapest, Hungary, July 2010.

[15] M. Klug, E. B. Castelan, and V. J. S. Leite. A dynamic compensator for parameter varying systems subject to actuator limitations applied to a T-S fuzzy system. In Proceedings of the 18th IFAC World Congress, pages 1449514500, 2011.

[16] J. Melin, M. Jungers, J. Daafouz, and C. Iung. Performance analysis and design of dynamic output feedback control for switched systems. International Journal of Control, 84(2):253-260, 2011.

[17] V. M. Moraes, E. B. Castelan, and U. F. Moreno. A dynamic output feedback compensator for time-stamped networked control systems. In Submitted to Brazilian Conference of Automatic Control, CBA, 2012.

[18] J. H. Park. On dynamic output feedback guaranteed cost control of uncertain discrete-delay systems: LMI optimization approach. Journal of Optimization Theory and Applications, 121(1):147-162, April 2004.

[19] I. R. Petersen. A stabilization algorithm for a class of uncertain linear systems. Systems $\&$ Control Letters, 8:351$357,1987$.

[20] J.P. Richard. Time delay systems: An overview of some recent advances and open problems. Automatica, 39(1):1667-1694, 2003.

[21] C. Scherer, P. Gahinet, and M. Chilali. Multiobjective output-feedback control via LMI optimization. IEEE Transactions on automatic control, 42(7):896-911, 1997.

[22] M. Tai and K. Uchida. Gain schedule output feedback of discrete-time networked control systems. In 16th IEEE International Conference on Control Applications. Singapore, 2007

[23] G. Walsh, H. Ye, and L. Bushnell. Stability analysis of network control systems. IEEE Transactions on Control Systems Technology, 10(3):438-446, 2002.

[24] W. Zhang, M. S. Branicky, and S. M. Phillips. Stability of networked control systems. IEEE Control Systems Magazine, pages 84-99, February 2001. 